
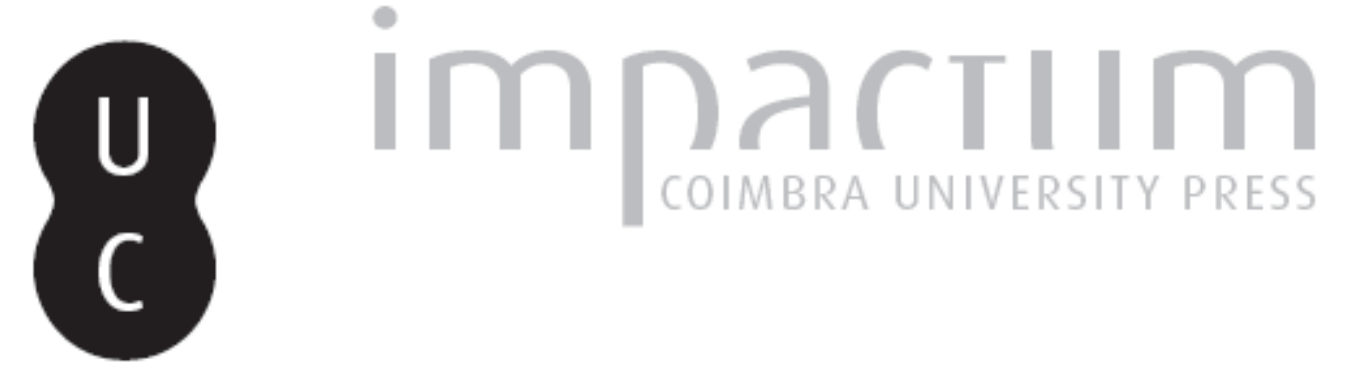

\title{
Análise comparativa sobre os estudos bioclimáticos no Brasil e em Portugal: articulações e desafios
}

Autor(es): $\quad$ Aleixo, Natacha; Sant'Anna Neto, João Lima; Cunha, Lúcio

Publicado por: $\begin{aligned} & \text { Faculdade de Letras da Universidade de Coimbra, Departamento de } \\ & \text { Geografia }\end{aligned}$

URL

persistente:

URI:http://hdl.handle.net/10316.2/30193

Accessed : $\quad$ 26-Apr-2023 04:19:23

A navegação consulta e descarregamento dos títulos inseridos nas Bibliotecas Digitais UC Digitalis, UC Pombalina e UC Impactum, pressupõem a aceitação plena e sem reservas dos Termos e Condições de Uso destas Bibliotecas Digitais, disponíveis em https://digitalis.uc.pt/pt-pt/termos.

Conforme exposto nos referidos Termos e Condições de Uso, o descarregamento de títulos de acesso restrito requer uma licença válida de autorização devendo o utilizador aceder ao(s) documento(s) a partir de um endereço de IP da instituição detentora da supramencionada licença.

Ao utilizador é apenas permitido o descarregamento para uso pessoal, pelo que o emprego do(s) título(s) descarregado(s) para outro fim, designadamente comercial, carece de autorização do respetivo autor ou editor da obra.

Na medida em que todas as obras da UC Digitalis se encontram protegidas pelo Código do Direito de Autor e Direitos Conexos e demais legislação aplicável, toda a cópia, parcial ou total, deste documento, nos casos em que é legalmente admitida, deverá conter ou fazer-se acompanhar por este aviso.

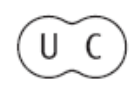




\title{
Análise comparativa sobre os estudos bioclimáticos no Brasil e em Portugal. Articulações e desafios
}

\author{
Natacha Aleixo \\ Universidade Estadual Paulista - UNESP campus Presidente Prudente. \\ natachaaleixo@yahoo.com.br
}

\section{João Lima Sant 'Anna Neto}

Universidade Estadual Paulista - UNESP campus Presidente Prudente. joaolima@fct.unesp.br

\section{Lúcio Cunha}

Departamento de Geografia e Centro de Estudos de Geografia e Ordenamento do Território (CEGOT). Faculdade de Letras da Universidade de Coimbra. luciogeo@fl.uc.pt

\section{Resumo:}

As diferenciações climáticas e a vulnerabilidade das populações em distintas dimensões espaço-temporais repercutem-se em situações diferenciadas de riscos socioambientais. Assim, o objetivo deste trabalho é analisar os riscos climáticos e os impactos na saúde nos territórios brasileiro e português, compreendendo as especificidades, as dinâmicas territoriais e as diferentes geografias que se verificam em ambos os países. Para isso, foram selecionadas pesquisas e estudos publicados sobre bioclimatologia humana em ambos os territórios e foram coletados dados da Organização Mundial da Saúde (WHo). Esses dados foram tratados estatisticamente para a compreensão das diferenças no perfil de morbilidade das populações brasileira e portuguesa, associadas aos riscos climáticos. Verificou-se que as condições de vulnerabilidade socioambiental e de gestão dos fatores de risco oferecem capacidade de resiliência diferenciadas nos territórios. Neste contexto, a utilização da climatologia como instrumento para o planeamento e promoção da saúde é fundamental para diminuição dos impactos.

Palavras-Chaves: Risco climático. Clima urbano. Saúde pública. Brasil. Portugal.

\section{Résumé:}

Analyse comparative sur les études bioclimatiques au Brésil et Portugal: relations et défis

Les différences climatiques et la vulnérabilité des populations dans les différentes dimensions spatio-temporelles entrent en résonance avec les différentes situations de risque pour l'environnement. L'objectif de cette étude est d'analyser les risques climatiques et les impacts sur la santé au Brésil et au Portugal, y compris les détails, les dynamiques territoriales et les zones géographiques différentes qui existent dans les deux pays. À cette fin, nous avons sélectionné la recherche et des études sur la bioclimatologie humaine dans les deux territoires, en plus, les données ont été recueillies auprès de l'Organisation mondiale de la Santé ( $\left.W_{H O}\right)$. Ces données ont été analysées statistiquement afin de comprendre les différences dans le profil de morbidité de la population brésilienne et portugaise, les risques associés climáticos. La vulnérabilité des conditions socio-environnementales et la gestion des facteurs de risque de fournir la résilience dans les différents territoires. Dans ce contexte, l'utilisation de la climatologie comme un outil pour la promotion de la planification et de la santé est essentielle pour l'atténuation des impacts.

Mots-Clés: Le risque climatique. Le climat Urbain. La santé publique. Le Brésil. Le Portugal.

\section{Abstract:}

Comparative analysis of bioclimatic studies in Brazil and Portugal: joints and challenges

The climatic differences and population vulnerability of distinct space-time dimensions reverberate in different situations of socio-environmental risk. Thereby, the objective of this study is to analyze climatic risks and impacts on health in Brazil and Portugal territory; comprising the specificities, territorial dynamics and the different geographies that exist in both countries. For this purpose, published research and studies on human bioclimatology on both territo- 
ries were selected, moreover, data from World Health Organization (WHO) was collected. This data has been statistically analyzed for the understanding of the morbidity profile differences between Brazilian and Portuguese population, associated to the climatic risks. It was verified that the vulnerability socio-environmental and risk factors management conditions offer differentiated resilience capacity in the territories. In this context, the use of climatology as an instrument for planning and promotion on health is essential for impacts reduction.

Keywords: Climatic risk. Urban climate. Public health. Brazil. Portugal.

Introdução

O processo saúde-doença tem relação direta com as condições do ambiente. Desde os primórdios de nossa civilização, com os estudos de Hipócrates, condensados na obra Dos ares, das águas, dos lugares, destacou-se o papel do ambiente na génese, determinação e agravamento das doenças, mas o caráter científico sobre essa temática foi evoluindo ao longo dos anos e, no contexto contemporâneo, incorporou as práticas socioculturais e a vulnerabilidade socioambiental na compreensão desta relação, que é interdisciplinar.

As inundações urbanas, a poluição do ar, as ondas de calor e de frio, bem como o desconforto térmico são alguns dos problemas que afetam a vida social e a saúde das populações, tornando-se campo de interesse da abordagem geográfica.

Os eventos atmosféricos extremos aliados à ausência de medidas de planeamento urbano e de contenção de inundações, além da disposição inadequada de resíduos e falta de saneamento básico, podem propiciar a génese de doenças relacionadas com a água. Por outro lado, os períodos de seca prolongada, a baixa humidade relativa do ar e o aumento dos poluentes na atmosfera constituem agravantes para a ocorrência das doenças respiratórias.

As diferenciações climáticas e os diversos matizes que caracterizam a vulnerabilidade das populações em distintas dimensões espaço-temporais propiciam situações diferenciadas de riscos socioambientais.

Neste contexto, é importante analisar que a localização e a produção de distintos territórios, como os de Portugal e do Brasil, com semelhanças e diferenças, auxilia na compreensão dos processos e especificidades que apresentam, bem como dos distintos níveis de desenvolvimento e desigualdades socioambientais, que condicionam novas organizações dos contextos físicos e humanos.

Assim, o objetivo deste trabalho é analisar os riscos climáticos e os impactos na saúde no território brasileiro e português, compreendendo as especificidades, as dinâmicas territoriais e as diferentes geografias de ambos os países.

\section{Procedimentos metodológicos}

Primeiramente, realizou-se uma revisão bibliográfica dos estudos luso-brasileiros, publicados sobre a relação clima e saúde humana, publicados em periódicos como a Revista Territorium (Portugal), Revista Portuguesa de Saúde Pública (Portugal), Revista Brasileira de Climatologia (Brasil), entre outras. Assim, identificaram-se os principais problemas de saúde pública potencializados pelos riscos climáticos, em ambos os territórios.

Além disso, foram coletados dados de morbilidade da Organização Mundial da Saúde (WHO). Esses dados foram tratados estatisticamente, para a compreensão das diferenças no perfil de morbilidade da população brasileira e portuguesa, associadas aos ris cos climáticos.

\section{O estudo da bioclimatologia humana}

O campo de estudo que relaciona as condições meteorológicas e a medicina é denominado Bioclimatologia e/ou Biometeorologia e os primeiros trabalhos publicados foram sobre a influência da precipitação in tensa nas doenças transmitidas por vetores (TROMP, 1980).

A atmosfera é parte do ambiente, pois, devido às mudanças de tempo, o organismo humano é forçado a adaptar-se termicamente para permanecer com saúde, com regulações independentes de comandos mecânicos dos indivíduos. No entanto, algumas pessoas possuem uma adaptabilidade mais sensível como acontece com idosos, doentes, mulheres grávidas e crianças (JENDRITZKY et al., 1994).

SORRE (1984) denominava essa parte da população de anemossensíveis, mas atualmente tem-se denominado como efeito meteorotrópico a ação que a diferente variabilidade do estado do tempo provoca sobre a saúde das pessoas.

Hoppe (1997: 19) relatou que as primeiras pesquisas científicas sobre biometeorologia começaram na primeira metade deste século. Na segunda metade deste século, os estudos focaram "as descrições quantitativas das trocas térmicas entre o corpo humano e o 
meio ambiente, por meio de modelos de equilíbrio energético que obtiveram importância crescente".

Para Meade, Florin e Gesler, citados por Ribeiro (1996:4), "a biometeorologia preocupa-se em esclarecer as variações e mudanças nos sistemas físico-químicos dos organismos vivos, em especial dos seres humanos, bastante sensíveis a alterações atmosféricas de diversas ordens: temperatura, humidade, ventos, pressão, radiação solar, poluição atmosférica, descarga elétrica, magnetismo, etc."

Os efeitos da atmosfera nunca estão isolados e na biometeorologia, segundo JENDRITZKY (1994), distinguemse três campos principais de atuação: as condições complexas da troca de calor do ser humano, a fim de manter o equilíbrio térmico, os fluxos de radiação de onda curta e longa e a poluição atmosférica.

A adaptação humana baseia-se nas características funcionais e estruturais da população conforme a condição ambiental a que está exposta. Os estudos tendem a enfatizar as múltiplas possibilidades de resposta do Ser Humano frente ao ambiente, ainda que existam diferenças com relação aos termos adaptabilidade e ajuste.

Para MORAn (1994:26) "formas extremas e constantes de stress podem ser enfrentadas por meio de uma alteração fisiológica irreversível durante o período de desenvolvimento do indivíduo (ajustes de desenvolvimento); por outro lado, as formas de aclimação de respostas fisiológicas facilitam o ajustamento dos indivíduos após o período de desenvolvimento e são reversíveis (ajustes de aclimação). As formas mais comuns e flexíveis de ajustamento, talvez sejam as comportamentais, sociais e culturais (ajustes reguladores)".

Por isso, as características de ajustamento e adaptabilidade humana variam de um indivíduo para outro nos diferentes locais do globo, bem como varia a capacidade de resposta por meio dos ajustamentos reguladores.

O grau de aclimatação do indivíduo é importante e tem sido modificado pelas alterações térmicas nos ambientes urbanos, criando uma nova atmosfera na camada limite planetária que afeta as condições de adaptação, com consequência na saúde dos seres humanos. Têm-se denominado os estudos da relação entre o clima urbano e a saúde humana como estudos de biometeorologia urbana.

Segundo SILVA (2010: 50) "a bioclimatologia humana aplicada às cidades relaciona-se com aspetos do clima urbano e sua influência no conforto e desconforto e na saúde humanos. Os efeitos do espaço urbano nos componentes do clima, tais como temperatura, humi- dade, radiação e vento, elementos importantes para a manutenção do balanço de calor do corpo humano têm sido documentados em todo o mundo. Desse modo, considera-se que, atualmente, há uma base de conhecimento para aplicação da avaliação biometeorológica e/ ou bioclimatológica nas áreas urbanas”.

De acordo com Gonçalves e Krieger (2004: 1), a biometeorologia urbana é o estudo biometeorológico do ambiente atmosférico de áreas urbanas em relação à saúde humana e à vegetação. Desde a Grécia Clássica e a Roma Antiga que a preocupação com os fenómenos biometeorológicos urbanos já existia, porém, somente a partir do século XIX estes estudos começam a ser tratados quantitativamente.

A escala de estudo da biometeorologia (dias, horas) e bioclimatologia (meses e anos) humana é, cada mais, a do ambiente urbano, pois as cidades têm-se tornado o lugar do viver da sociedade e a alteração dos componentes físicos nessa escala repercutem-se diretamente na saúde. Os impactos na vida social ocorrem de forma exacerbada com os processos de expansão urbana desordenada, como por exemplo, a construção das moradias nos ambientes endémicos de vetores que transmitem doenças como a leishimaniose, malária, doença de hagas, entre outras.

Com a alteração na quantidade de matéria e energia do sistema climático, provocada pela produção e expansão dos espaços urbanos, as condições normais de saúde-doença, bem como de sobrevivência dos vetores podem ser alteradas, consequentemente, a capacidade de adaptação e stress humano também.

JAUREGUI (1994) analisou o desenvolvimento de estudos de clima urbano nos trópicos a partir de informações de periódicos científicos e jornais, desde a década de 70 até 90 e verificou que ocorreu um aumento de publicações durante a década de 90 comparada com as anteriores, bem como um aumento de pesquisas relacionadas com a bioclimatologia urbana, o conforto térmico e a saúde.

Entretanto, o aumento na produção de estudos biometeorológicos não está diretamente associado à sua qualidade, pois, DrIscoll (1999), a partir de uma revisão de estudos publicados na década de 90 pelo principal periódico científico do tema, o Internacional Journal of Biometeorology, verificou que a produção em termos de biometeorologia humana quantitativa tinha de ser entendida à luz das limitações dos métodos estatísticos. 0 autor verificou uma relação de causa e efeitos incompleta nos trabalhos, pois os métodos estatísticos empregados (correlação, regressão, análise do qui-quadrado, variância, assim como outras técnicas 
mais sofisticadas), apenas servem para investigar a validade matemática de uma presumível relação causa e efeito. Entretanto, muitas vezes, o conhecimento da etiologia da doença é desconhecido e/ou ainda incompleto na medicina.

Além de se comprovar uma exagerada influência da sazonalidade com correlações significativas, "it is clear from the concluding remarks of some studies that the author expected to find significant associations. Because they weren't found, as sometimes happens, he or she adopts an apologetic tone - almost a defensive posture and tries to rationalize this negative findings. It seems to me that it is the finding of significant associations that must be rationalized, not their absence. The weather and cosmos are innocent until proven guilty or at least until the evidence becomes preponderant" (DrISCOLL, 1999:75).

Neste campo de estudo interdisciplinar, o autor diz-se esperançado com a união de profissionais que investigam o ambiente e a medicina prática, pois, sendo "a doença um produto biológico e das forças culturais, esta pode ser compreendida em quatro etapas: as condições do ambiente, a resposta do organismo ou sistema, os indicativos ou manifestações fisiológicas e a deficiência ou modificação da capacidade adaptativa”. (DRISCOLL, 1999:76)

No Brasil, Galvani (2005:5691) analisou os Anais do Simpósio Brasileiro de Biometeorologia, desde a primeira edição em 1996, ordenando os trabalhos em "estudos bioclimáticos, que apresentam aspetos analíticos em uma escala de tempo maior, e estudos biometeorológicos, que apresentam os efeitos imediatos do tempo nos seres vivos". Assim, o autor pôde concluir que a maior parte dos estudos são de bioclimatologia, por isso, a própria sociedade brasileira de biometeorologia poderia mudar o nome, para representar a produção gerada pelos sócios.

Pela grande diversidade de pesquisas ligadas a diferentes áreas no III Simpósio Brasileiro de Biometeorologia, foram norteados três eixos temáticos: Humano, Animal e Vegetal. Assim, para Galvani (2005:5607), "os estudos devem ser considerados em suas análises específicas do clima de cada região representando um quadro mais real das relações entre os seres vivos e os elementos do clima".

Nas pesquisas científicas a difusão de temas envolvendo as condições climáticas e meteorológicas no processo saúde-doença, tem requerido novas abordagens, pois a complexidade dos problemas não é mais passível de ser analisada à luz dos conceitos e técnicas desenvolvidas por uma única ciência. É preciso tentar englobar conhecimentos de outras ciências buscando a interdisciplinaridade.

A capacidade de adaptação dos seres humanos aos diferentes tipos de tempo é ampla, porém, a facilidade ou dificuldade dessas adaptações pode ser alterada pelas diferentes vulnerabilidades e capacidades de resiliência da população. Por isso, o entendimento das características climáticas e das condições de vida da população é objeto para estudos de diferentes campos do conhecimento.

O próprio conceito discutido (biometeorologia e/ ou bioclimatologia humana) corresponde a um ramo do conhecimento da Saúde Pública bem como de diversas outras ciências como a Geografia, a Meteorologia, a Agronomia, a Medicina, a Arquitetura e a Física.

$\mathrm{Na}$ biometeorologia e/ou bioclimatologia podese identificar a relação do clima com o processo saúdedoença de maneira qualitativa e quantitativa. A influência das condições higrotérmicas do ambiente pode ser avaliada por meio de entrevistas sobre a perceção do conforto, as sensações experimentadas e as condutas utilizadas.

Ao verificar quantitativamente esta relação, são utilizados questionários e/ou técnicas estatísticas sofis ticadas, que evidenciam desfasagens de dias ou, mesmo, de meses na correlação de casos das doenças e tipos de tempo e/ou eventos climáticos. Ainda assim, é difícil avaliar os efeitos climáticos na saúde e separar os fatores de confusão como os determinantes socioeconómicos, culturais e comportamentais.

O campo de conhecimento da biometeorologia e/ ou bioclimatologia é claramente interdisciplinar, mas, muitas vezes, os profissionais absolutamente competentes no âmbito da sua formação, não utilizam de todas as técnicas possíveis para o entendimento do problema, pela falta de alguns conhecimentos técnicos ou teóricos que não foram apreendidos no seu campo de formação.

Os profissionais avançam no desafio de tentar entender a totalidade do problema em estudo, que supera os conhecimentos de uma disciplina ou mesmo da ciência de formação. A complexidade estudada neste campo de conhecimento merece respeito pelo reconhecimento da totalidade pelos profissionais, ainda que, na prática, estes não consigam incorporar as múltiplas facetas envolvidas no objeto de estudo. Por outro lado, a abordagem a partir de apenas uma linha de conhecimento não alcançaria a profundidade necessária para se compreender a problemática em toda a sua complexidade e totalidade.

Como esta pesquisa se insere na ciência geográfica, pode-se verificar as suas ramificações na geografia 


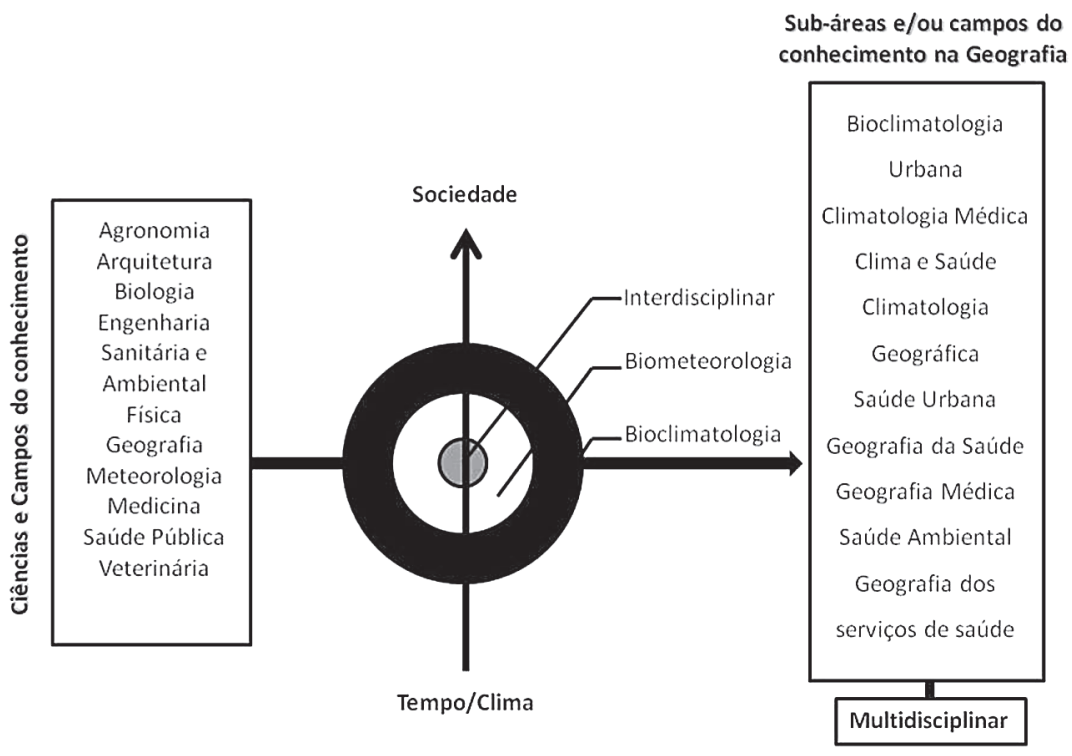

Figura 1

Subáreas e/ou campos do conhecimento bioclimatológico e biometeorológico. Elaboração: ALEIXo (2011).

da saúde, geografia médica, geografia dos serviços de saúde, climatologia geográfica, entre outros, conforme a Figura 1.

Neste contexto, acredita-se que a principal contribuição do geógrafo que trabalha com este tema é a investigação da distribuição espacial do fenómeno, da relação com as condições socioambientais vividas nos espaços e lugares (entendendo a dinâmica atmosférica como uma parte deste complexo socioambiental), da organização dos serviços de saúde e da perceção da população acerca da causa da doença e do seu comportamento preventivo.

A especificação do estudo biometeorológico e/ou bioclimatológico em Geografia faz com que os pesquisadores utilizem as perspetivas produzidas pelas sub-áreas e/ou campos do conhecimento, porém, devem ter como princípio a totalidade do problema, ou seja, integrar e não segmentar ainda mais.

No Brasil, os primeiros estudos de bioclimatologia humana foram realizados nas pesquisas das denominadas "doenças tropicais" (cólera, malária, doença do sono, etc.), para compreender a etiologia das doenças e a forma como elas se manifestam no território devido ao clima. Essa denominação estava ligada à colonização, reconhecimento e ocupação do território brasileiro pelos europeus e foi responsável pelos primeiros estudos de clima e saúde produzidos no Brasil por médicos e sanitaristas.
Além do ambiente: o espaço produzido e as doenças tropicais no Brasil

No Brasil, as primeiras impressões sobre o tempo e o clima advêm dos viajantes naturalistas, mas o estudo da climatologia interessava principalmente pela determinação que cabia ao clima na gênese das doenças denominadas tropicais (SANT'ANNA Neto, 2003).

Até ao século XIX, a expansão colonial foi um fator importante para fundamentar a "culpa" climática e as antigas noções de que as altas temperaturas e o calor excessivo diminuíam a força e a coragem dos homens (Peixoto, 1938; Albuquerque et al., 1999: 424).

"As doenças nos países frios foram sempre consideradas uma fatalidade independente do clima, não se Ihes sabia a causa, mas outras eram as suposições dos patologistas. Para países quentes, porém, o critério é diverso: sem mais exame, o clima é apontado como causa maior, junto da qual são todas menos importantes" (Реіхото, 1938:154).

A primeira matriz de expansão científica desse tema advém da época dos descobrimentos e da conquista e colonização dos territórios. A Europa traz um modelo difusionista de produção das pesquisas científicas pelo mundo, pautada na expansão da europeização pelos viajantes e naturalistas, que têm o propósito de inventariar e catalogar o "novo mundo", "os territórios 
conquistados", até ao século XV inexistentes para os europeus (BASALLA, 1997).

As doenças tropicais são assim chamadas, pois a sua etiologia é condicionada por fatores que ocorrem nos trópicos, como altas temperaturas e humidade, abundância de precipitação no Verão, que propiciam o aumento de zoonoses e o desenvolvimento entomológico de vetores de algumas doenças.

Além dos fatores climáticos, as condições biogeográficas também influenciam a manifestação das zoonoses tropicais.

Concorda-se com Barros (2006) em que o termo "doenças tropicais e exóticas" esteja impregnado de preconceito pela herança da mentalidade europeia. 0 autor procurou compreender que, aos olhos de quem chega (estrangeiro), existe preconceito e aversão, um desapego aparente que se intensifica ou desaparece desde a colonização até ao século XX.

Relacionando a abordagem literária brasileira como Grande Sertão Veredas, Sagarana, Jeca Tatu, que apresentou algumas doenças tropicais, BARROS (2006: 299) relatou que o trópico é a soma de tudo, ou seja, ao mesmo tempo que é o paraíso perdido, é também a barriga vazia de comida e cheia de verminose e o queixo preguiçoso.

Camargo (2008: 96), ao analisar as diferentes etiologias das doenças tropicais, faz uma ressalva importante: "todas as doenças humanas, em princípio, são tropicais, uma vez que a espécie humana se originou nos trópicos e, com ela, suas doenças. São exceção aquelas doenças que a humanidade foi adquirindo ao longo de sua história (algumas recentemente) de companheiros de jornada como cães, gatos, roedores, aves e mesmo de parentes próximos, os primatas".

Na denominação "doenças tropicais" não são levados em conta alguns fatores que potencializam a ocorrência dessas doenças, como a condição socioeconómica da população para o enfrentamento das mesmas, o acesso aos serviços de saúde, a informação para prevenção e a capacidade de resiliência às epidemias recorrentes.

As doenças tropicais são intituladas como se ocorressem exclusivamente nos trópicos e a área geográfica denominada climaticamente como tropical é considerada com extensão e características homogêneas. Entretanto, existem inúmeros sub-trópicos, dependendo da formação geológica, geomorfológica e biogeográfica. Os ecossistemas diferenciados produzem biodiversidades singulares. (CAMARGO, 2008)

Mas, o que têm os trópicos de homogéneo?

Historicamente, o processo colonial de exploração das metrópoles europeias. No âmbito socioeconó- mico nenhum outro aspeto. Apesar da globalização da economia, os impactos da relação global-local e a inserção dos países no mercado mundial são diferentes. Pode-se dizer que existem inúmeras condições de desigualdade dentro dos trópicos, se comparados entre si.

Apesar de terem o clima quente e húmido e o inverno geralmente ameno, ainda assim são heterogéneos em vários aspetos, que impossibilitam uma lei geral sobre os mesmos (CAMARGo, 2008).

Com a degradação ambiental dos ecossistemas tropicais, nos últimos anos ocorreu diminuição e readaptação da composição das espécies e a rutura de processos ecológicos, consequentemente, a desestabilização dos ciclos ecológicos de transmissão dos vetores das patologias tropicais e a modificação dos padrões considerados, como entendidos anteriormente.

Pode-se dizer que ocorreu uma polissemia do conceito, que foi relacionado ao determinismo, ao colonialismo, à falta de higiene e, atualmente, à pobreza e à desigualdade social (Albuquerque, 1999; Barros, 2006; Camargo, 2008; Peixoto, 1938).

A existência de amplo conhecimento sistematizado sobre as doenças tropicais, desde a época colonial e, posteriormente, "a institucionalização da medicina tropical como disciplina no final do século XIX, auxiliaram a mudar as conceções que priorizavam os fatores ambientais como única causa das doenças tropicais" (MoRAIs, 2007: 60).

$\mathrm{Na}$ era pasteuriana, com o avanço no controle da microbiologia e no controle de vetores, "os fatores climáticos passaram de causa eficiente para causa predisponente" (MoRAIs, 2007: 60). Entretanto, a denominação de doença tropical se manteve, ainda que permeada com outras perspetivas (ForatinNI, 1997).

Concordamos com Albuquerque et al. (1999: 430) quando os autores afirmam que a "visão fatalista dos trópicos, cujo principal componente foi a determinação climática, está hoje minimizada pela noção de que as doenças são sociais, economicamente impostas e ecologicamente ambientadas".

As doenças tropicais no século XX foram analisadas pela ótica económica e percebeu-se que a pobreza está vinculada diretamente com a permanência delas nos países tropicais. Chegou a escrever-se o termo "doenças do subdesenvolvimento", mas atualmente têm-se denominado "doenças tropicais negligenciadas", sendo o termo adotado pela OMS, com a conceção da questão socioeconómica, deixando de colocar a dependência da causa, apenas nos trópicos.

Mas as doenças tropicais são negligenciadas por quem? 
Conforme Villa (2009: 437) "um sistema de falhas ocorre e faz com que as patologias persistam no Brasil: falha de ciência (conhecimentos insuficientes); falha de mercado (alto custo de medicamentos ou vacinas); falha de saúde pública (planeamento deficiente para diagnósticos e tratamentos). As falhas de ciência exigem uma reorganização do sistema nacional de pesquisa, considerando a construção de uma ponte entre a produção do conhecimento e sua aplicação nos vários setores, as falhas de mercado requerem mecanismos inovadores de financiamento ou negociações, as falhas de saúde pública exigem novas estratégias de intervenção".

Acrescentam-se a estas, as falhas na previsão, na monitorização e no alerta dos serviços meteorológicos que minimizariam a parte que cabe ao clima tropical como condicionante dessas doenças, ainda que o princípio da incerteza esteja sempre presente.

A análise da produção e da apropriação do espaço, bem como as doenças tropicais desatendidas e/ou negligenciadas podem ser explicadas sociohistoricamente como uma função da globalização no espaçotempo.

A OMS (2011) define as doenças tropicais negligenciadas como "doenças infecciosas que prosperam em ambientes pobres, no calor e humidade do clima tropical. Na maioria são doenças parasitárias, transmitidas por insetos ou transmitidas por água contaminada e solo infestado com ovos de vermes. Os ciclos de transmissão são perpetuados em condições de contaminação e degradação ambiental que, por sua vez, são perpetuadas pelos padrões pobres de vida e higiene. Outrora dispersas, estas doenças estão agora concentradas em locais de extrema pobreza, nas favelas urbanas ou em zonas de conflito e desenvolvem-se em condições de empobrecimento. Em grande parte do mundo, estas doenças desapareceram gradualmente com a melhoria dos padrões de vida e higiene" (World Health OrganizaTION, 2011: 1).

O grupo das doenças tropicais negligenciadas é composto por dezassete doenças: Úlcera de Buruli, Leishmaniose, Doença de Chagas, Lepra, Cólera, Doença diarreica epidémica, Filaríase linfática, Dengue/Febre hemorrágica de dengue, Oncocercose, Dracunculíase, Schistossomose, Treponematose endémica, Helmintoses transmitidas pelo solo, Tracoma, Tripanossomíase humana africana, Tuberculose, HIV/SIDA e Malária.

É contraditório observar como o progresso científico brasileiro pouco tem tentado preencher as lacunas dos problemas de saúde pública e as epidemias das doenças tropicais negligenciadas. Para ForatTINI (1997: 119) "sem pretender negar a utilidade do desenvolvi- mento tecnológico, no entanto, há de se ponderar que os nossos pesquisadores comportam-se como se a população brasileira não mais tivesse de se defrontar com os problemas constituídos pelas endemias e infeções emergentes".

Como exemplo, o autor refere-se à doença de Chagas, considerada uma das patologias de ampla distribuição no continente americano, que completou, em 2009, cem anos de seu descobrimento.

"Pensar uma sociedade livre de doenças devido ao desenvolvimento social e tecnológico é ilusório", pois nem os países subdesenvolvidos nem os desenvolvidos têm o foco das suas preocupações nos problemas de saúde, mas sim na produção económica e no aumento do PIB (Confalonieri, 2002: 54).

O processo saúde-doença e as desigualdades em saúde ocorrem e abrangem uma escala maior que vai além do status de desenvolvimento adquirido. Assim, a pergunta a que os pesquisadores tentam responder é se no caso das chamadas "doenças tropicais" o fatalismo tropical pode ser combatido com desenvolvimento económico e sanitário? Ainda ocorreriam pandemias das chamadas doenças tropicais negligenciadas?

Uma boa dose de desenvolvimento, a melhoria e a oferta de condições de saneamento básico são importantes. Entretanto há que considerar a produção da saúde a partir dos seus problemas, ou seja, condizente com os interesses da população e enriquecendo-lhes a cultura.

Por isso, os trabalhos em microescala, ou seja, no local, transitando pelas outras escalas analíticas do processo saúde-doença, são importantes para verificar os condicionantes que influenciam na permanência e emergência das patologias tropicais.

As cidades, como produto de um processo de urbanização desordenada e planeada de forma inadequada, são responsáveis pela flexibilização das relações que possibilitam maior mobilidade e circulação de agentes, vetores, aumento da população e do consumo de produtos com embalagens biodegradáveis e urbanidade de doenças, como a leishimaniose e a dengue.

Para além de, no Brasil, algumas das denominadas "doenças tropicais" terem alta incidência nos dias de hoje, o número de casos de doenças cardiovasculares e respiratórias relacionadas com os tempos rápidos da vida moderna, as ilhas de calor e a poluição do ar nos centros urbanos, também aumentou significativamente.

No contexto da Geografia brasileira, muitos estudos foram realizados no estado de São Paulo, com destaque para o trabalho de RibeIRo SOBRAL (1988) sobre a relação da poluição do ar e doenças respiratórias em 
São Paulo. A autora demonstrou o aumento de sintomas de problemas respiratórios em crianças nas áreas mais poluídas da metrópole paulistana.

Na mesma cidade, SıLva (2010) verificou a relação do índice de conforto Pet com a ocorrência de doenças cardiovasculares e respiratórias. A autora identificou que as condições de desconforto para o frio e a elevada amplitude térmica, constituiam fatores agravantes para o desencadeamento das doenças.

A associação de crises hipertensivas e cardíacas com tipos de tempo agravantes foi estudada por PITTON e Domingos (2004). Além desse estudo, na cidade de Presidente Prudente/SP, Grosso (2007) e Murara (2010) verificaram a relação dos sistemas atmosféricos e dos elementos climáticos com a ocorrência de casos de doenças respiratórias e óbitos por doenças circulatórias.

Além desses, na região Sul do país, MEndonçA (2000, 2002, 2006) estudou a expansão da dengue no Sul do Brasil e discutiu a repercussão das mudanças climáticas na diminuição do tempo de desenvolvimento do vetor e possível aumento de casos da doença. FerrelRA e LOMBARDo (2000) avaliaram a ocorrência de malária na área de influência do reservatório de Itaipu.

Nas cidades brasileiras, principalmente nas do interior do estado de São Paulo, além da queima de combustíveis fósseis, outros fatores contribuem para o aumento da poluição do ar, sendo um deles a queima da palha da cana-de-açúcar que lança uma enorme quantidade de produtos gasosos potencialmente prejudiciais ao aparelho respiratório.

Os efeitos das queimadas na saúde foram pouco estudados até o presente momento, pois, de acordo com Ribeiro e Assunção (2002), existe muita dificuldade de separar os fatores de confusão, como a influência dos tipos de tempo que dispersam ou não os poluentes liberados na queima, o tipo de poluente liberado e sua concentração, as diferentes condições socioeconómicas da população exposta, a suscetibilidade ou não à morbilidade (riníticos, cardiopatas, idosos e crianças), entre outros.

$\mathrm{Na}$ cidade de Ourinhos, a emissão de poluentes decorrentes da prática da queima da palha de cana-deaçúcar, de acordo com Aleixo (2008) colaborou no aumento dos casos de morbidade do aparelho respiratório, principalmente em indivíduos com predisposição (crianças, idosos, riníticos, entre outros) para patologias respiratórias. Esse aumento foi verificado quando se correlacionaram as condições meteorológicas à escala local com episódios de alta concentração de material particulado 2.5 e monóxido de carbono, na baixa troposfera.

Esses são alguns dos estudos ligados a temática que foram realizados no estado de São Paulo/Brasil e que comprovam a inter-relação dos tipos de tempo agravantes e do desordenamento do território como fatores de risco para a saúde humana.

\section{O clima e a saúde no contexto português: exemplos a diferentes escalas}

Diferente do Brasil, Portugal não tem problemas com as denominadas doenças tropicais ou negligenciadas, ainda que algumas delas ocorram no território, como a tuberculose, a leishimaniose, a doença diarreica, a par com casos importados da malária.

No contexto português, os riscos climáticos como as ondas de frio e calor, traduzem-se, basicamente, no aumento de casos de doenças respiratórias e cardiovas culares, atingindo principalmente a população idosa, que no território português corresponde a uma parcela muito significativa da população.

Um dos principais riscos climáticos associados à saúde da população são as ondas de calor, uma vez que no território português, pela adaptabilidade ao clima mediterrâneo e pelos invernos rigorosos na porção Norte do país, uma parcela significativa da população não possui sistema de arrefecimento nas casas, nem muitos lares de terceira idade, centros de saúde e hospitais o têm instalado.

As ondas de calor são fenómenos climáticos esporádicos, mas recorrentes, caracterizados por períodos de calor intenso com duração de vários dias e associadas ao aumento da mortalidade e da morbilidade (CUNHA, 2012:). A principal causa de morte atribuível é o golpe de calor, mas a onda de calor provoca um aumento da mortalidade por todas as causas, sobretudo por doença cardiovascular e respiratória (MENDES, 2009: 45). De acordo com MENDES (2009), a onda de calor do ano de 2003 terá causado 1953 óbitos no país.

Embora o sistema de saúde português tenha estabelecido melhorias nos sistemas de prevenção a esse risco climático, como é o caso do Plano de Contingência para as Ondas de Calor (PCOC), o qual funciona anualmente entre Maio e Setembro, estima-se que Portugal é o país da Europa com menor utilização de ar condicionado, o que acentua principalmente o risco de morbidade e mortalidade na população idosa que vive isolada.

Na região Centro de Portugal, 94\% dos hospitais possuem aquecimento e apenas $76,5 \%$ arrefecimento, sendo que a INSA revelou que em 2003, nos hospitais sem arrefecimento o número de mortos aumentou $60 \%$ (MENDES, 2009). 
Outro risco climático associado indiretamente com a saúde são os incêndios florestais, cujo impacto da fumaça e dos gases tóxicos podem potencializar os problemas de saúde na população portuguesa.

Os incêndios florestais ocorrem, muitas vezes, próximo aos espaços urbanos, pela expansão territorial urbana em meio florestal. Com essa ocupação e expansão desordenada, os particulados e produtos gasosos podem atingir mais facilmente a saúde respiratória da população.

0 risco de incêndios florestais é alto no país, devido ao clima mediterrâneo, que durante o verão oferece condições de alta temperatura e baixa umidade do ar, que ressecam a vegetação e podem criar condições favoráveis à ocorrência de incêndios (LoUREnço et al. 2001).

Segundo LoURenço et al. (2001), os factores físicos e humanos contribuem para o entendimento das condições do risco e combate aos incêndios florestais em Portugal.

GonÇALVEs et al. (2007:82) investigaram as causas de incêndios florestais dos anos de 1993 a 2003 e inferiram que "comportamentos negligentes e atitudes intencionais foram responsáveis por 66\% das ocorrências verificadas em Portugal Continental. Apenas, 2,5\% das ignições se teriam iniciado em relâmpagos e em 31,6\% dos casos não se conseguiu determinar a causa”.

Além disso, no período sazonal do verão, o desconforto térmico acentuado pelas ithas de calor nos centros urbanos também é responsável por situação de stress fisiológico, agravamento de doenças cardiovasculares e transtornos psico-sociais.

MARQUes e ANTUNES (2009:59) verificaram correlações elevadas entre a mortalidade e as temperaturas máximas, mínimas e médias no verão, mostrando que a ocorrência da "mortalidade respiratória nessa época do ano é influenciada, tanto por temperatura diurnas, como noturnas".

A influência da vulnerabilidade ao calor extremo também foi discutida por ANDRADE et al. (2011) que desenvolveram um modelo de vulnerabilidade da população da área metropolitana de Lisboa em relação ao calor, com base nos óbitos diários nas freguesias, na temperatura e em outros indicadores de vulnerabilidade.

Outro importante centro urbano do país, o Porto, tem sido estudado por Monteiro (1993, 1997 e 2001:865), que analisando crianças asmáticas acima de 5 anos, verificou que as maiores crises asmáticas ocorrem nos dias de "grande variabilidade térmica, ausência de precipitação, com velocidade do vento superior ao habitual e presença de situações de estabilidade atmosférica (an- ticiclone ibero-mediterrâneo e atlântico subtropical) de circulação zonal em altitude, com elevadas concentrações de poluentes na baixa atmosfera”. Além disso, o aumento do gradiente térmico da ilha de calor no Porto, associado à poluição do ar tem agravado os casos de crises asmáticas.

No inverno português, outro risco climático ocorre pela diminuição das temperaturas a valores extremos, que proporcionam condições para o aumento dos casos de doenças respiratórias e doenças cardiovasculares. As ondas de frio também são responsáveis pelo aumento da morbilidade e mortalidade desses grupos de patologias, em proporções significativas durante todos os anos.

$\mathrm{Na}$ cidade do Porto, por exemplo, o estudo de CARVAlHo (2007) diagnosticou que, no período de 1970 a 2005, o número de ondas de frio foi superior às ondas de calor.

Ressalta-se que as doenças cardiovasculares podem ser agravadas por valores extremos de temperatura máxima e mínima, dependendo das condições de vulnerabilidade da população, da menor capacidade de adaptação bioclimática em idosos e crianças, além de considerar o tipo de material construtivo da residência, a condição socioeconómica para utilização de equipamentos como aquecedores, bem como a perceção climática e o vestuário utilizado.

ALMENDRA (2010) verificou que durante o inverno, ocorre um excesso de internamentos, nos meses de novembro a março. Este acréscimo faz-se sentir em ambos os géneros e em todas as classes etárias analisadas, no entanto, é mais evidente na população idosa. As principais massas de ar associadas aos aumentos nos internamentos hospitalares por EAM, possuem características anticiclónicas, são secas e muito frias.

No país, embora o risco de inundações seja periódico, não foram encontrados estudos sobre a associação com problemas ou agravamento de saúde pública, demonstrando a pouca expressão desses episódios relacionados com doenças relacionadas com a água.

Ainda que a hipótese seja negativa, acredita-se que poderia também ser preenchida essa lacuna, com a realização de pesquisas para verificar as condições de vulnerabilidade dos moradores das áreas inundáveis e o impacto sobre a saúde humana.

\section{Articulações das escalas de análise}

Conforme a WHO (2002), não se pode eliminar o risco por completo, pois, não existe risco zero. Os indi- 
víduos e os lugares percebem as ameaças de diferentes formas, conforme as suas práticas e condições culturais e socioeconómicas. Neste contexto, a saúde e o ambiente não podem ser desvinculados, pois os riscos se materializam no ambiente e podem afetar a todos de diferentes maneiras.

Os riscos podem ser classificados em dois grupos: naturais e antrópicos. 0 primeiro acontece quando o fenómeno que produz danos tem a sua origem em processos da natureza, o segundo quando a origem ocorre por ações humanas.

No entanto, os fatores naturais, dentre eles os que representam o risco climático (ventos, chuvas intensas, geadas, ondas de calor, ondas de frio, incêndios, tempestades e secas prolongadas) quando em interação com as ações antrópicas no ambiente urbano tornaram-se mais complexos, pela intensificação do processo de urbanização e pela produção e apropriação do espaço de forma desordenada e desigual.

Dessa forma, o estudo dos riscos climáticos passa de um simples processo de monitorização e observação, para o entendimento social da produção do espaço, da vulnerabilidade e da resiliência da população. Apenas neste contexto, a prevenção dos riscos pode ser eficaz.

Os eventos climáticos extremos podem provocar e/ou potencializar uma série de riscos na saúde, dependendo do local onde ocorrem e da condição de enfrentamento da sociedade. No entanto, as chuvas e o aumento da temperatura do ar, oferecem condições importantes para o desenvolvimento dos vetores de doenças, como a dengue.

A conexão entre os riscos naturais e antrópicos pode ser contextualizada na ocorrência de fenómenos como as inundações, por exemplo, que de acordo com Rebelo (2001), pode ser considerada um risco climatico e hidrológico com impacto nos habitantes que ocupam e vivem nas áreas de várzea. 0 reflexo direto dessa articulação é a maior probabilidade de ocorrência de stress aos moradores, além dos efeitos indiretos, como a possibilidade de transmissão das doenças relacionadas com a água.

As doenças relacionadas com a água são bons indicadores da desigualdade em saúde na população, pois manifestam-se com maior intensidade nas áreas de

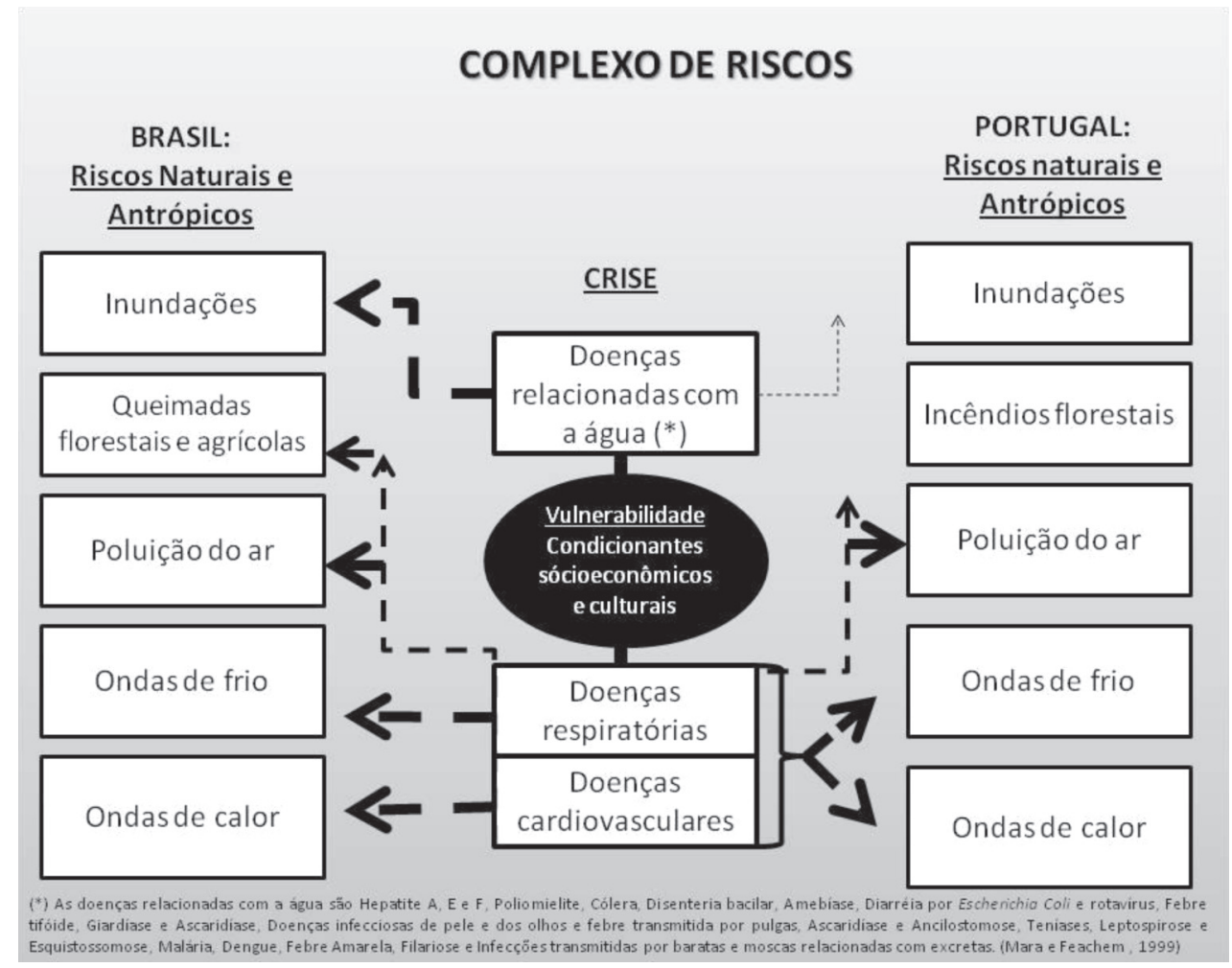

Figura 2

Complexo de riscos (Brasil e Portugal).

Organização: AleIXo (2012) 
Quadro I

Perfil de saúde: Brasil e Portugal (2009).

\begin{tabular}{|c|c|c|c|c|c|c|}
\hline Perfil de saúde & $\begin{array}{c}\text { Total da } \\
\text { população } \\
\text { (1000 hab.) }\end{array}$ & $\begin{array}{c}\text { População } \\
\text { urbana \% }\end{array}$ & $\begin{array}{c}\text { Rendimento Nacional } \\
\text { Bruto Per Capita }\end{array}$ & $\begin{array}{c}\text { Expectativa de vida } \\
\text { (anos) }\end{array}$ & $\begin{array}{c}\text { Taxa de mortalidade } \\
\text { menores de 5 anos } \\
\text { (100000/hab.) }\end{array}$ & $\begin{array}{c}\text { Taxa de mortalidade } \\
\text { infantil (100000/hab.) }\end{array}$ \\
\hline Brasil & 193734 & 86 & 10160 & 73 & 21 & 58 \\
\hline Portugal & 10707 & 60 & 24080 & 79 & 4 & 7 \\
\hline
\end{tabular}

Fonte: WHO (2011).

maior vulnerabilidade socioambiental, com escassez de programas preventivos.

Neste contexto, torna-se importante resumir esse complexo de riscos que afetam diretamente a saúde humana da população de ambos os territórios. Falase em complexo de riscos na Figura 2, para deixar claro os múltiplos condicionantes que perpassam o processo saúde-doença, ainda que a discussão deste trabalho enfoque o clima e seus tipos de tempo agravantes relacionados com a ocorrência das doenças respiratórias, cardiovasculares e relacionadas com a água.

As principais patologias que ocorrem no território português são as doenças cardiovasculares e respiratórias devidas aos intensos episódios de ondas de frio e calor. Além disso, os episódios de poluição do ar nos centros urbanos de porte grande do país, como Porto e Lisboa, também potencializam a ocorrência dessas patologias.

O território português apresenta poucos casos de doenças relacionadas com a água, dentre elas, a leptospirose, diarreias e casos importados de malária, mas que não estão associados diretamente aos riscos das inundações urbanas, como no caso brasileiro, em que os casos de leptospirose, ocorrem em maior intensidade na região Sudeste (principalmente nas metrópoles São Paulo e Rio de Janeiro) que apresentam durante a primavera-verão episódios frequentes de inundações nas áreas urbanas.

Os incêndios florestais apesar de ocorreram em todas as regiões portuguesas, nos últimos anos não causaram grandes problemas de saúde pública relativos ao aumento exacerbado de casos nos grupos dessas patologias, de acordo com os estudos consultados.

No Brasil, os incêndios florestais, por vezes chamados de queimadas, na sua maior parte ocorrem para desmatar a área, no intuito de utilizá-la para práticas agrícolas. Essas queimadas geram enormes quantidades de poluentes na atmosfera, que comprometem a saúde dos citadinos que moram no entorno dessas áreas.

Mas será, então, o clima brasileiro o responsável pela ocorrência diversificada destes grupos de patologias?
A resposta é, claramente, um não! Apesar das condições climáticas favorecerem a sobrevivência de alguns vetores transmissores de doenças, as condições de vulnerabilidade correspondentes a desigualdade social no país, ou seja, o espaço produzido, sentido e consumido de forma diferente pelas pessoas, beneficiando um pequeno grupo social, exacerba o risco.

Os indicadores compostos dos recenseamentos de ambos os países demonstram que em Portugal têm-se condições de maior qualidade de vida, que podem ser vistas no Quadro I, pela maior expectativa de vida, menores taxas de mortalidade infantil e prevalência de tuberculose, doença cuja incidência continua vinculada a condições socioeconómicas precárias.

Apesar do crescimento económico brasileiro, o país continua aquém na satisfação das condições sociais básicas, como, por exemplo, o acesso e qualidade da educação, saúde e saneamento. Além disso, as taxas de mortalidade de crianças menores de 5 anos dos dois países, refletem o perfil epidemiológico distinto, como se observa no gráfico 1 , pois, além das causas relacionadas com o nascimento e com o primeiro ano de vida, das que apresentam relação com o ambiente vivido, merecem destaque as taxas de pneumonia, diarreia e lesões, que são maiores no Brasil.

Gráfico 1

Taxa de mortalidade em crianças menores que 5 anos no Brasil e em Portugal no ano de 2008.

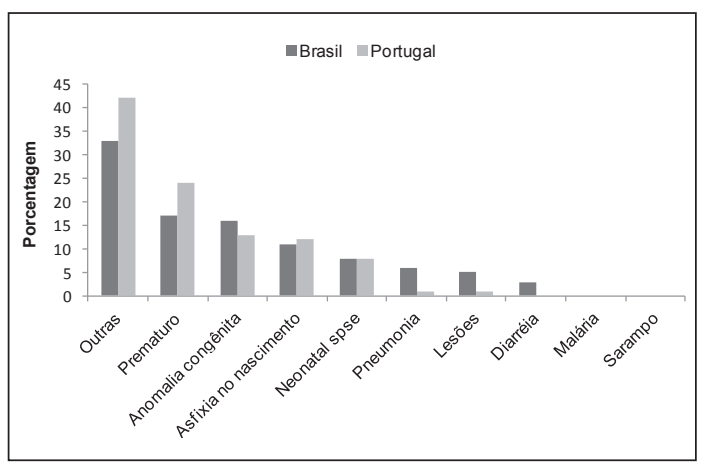

Fonte: Who (2011). 
Gráficos 2 e 3

Abastecimento de água potável e serviços sanitários no Brasil e em Portugal de 1990 a 2010.

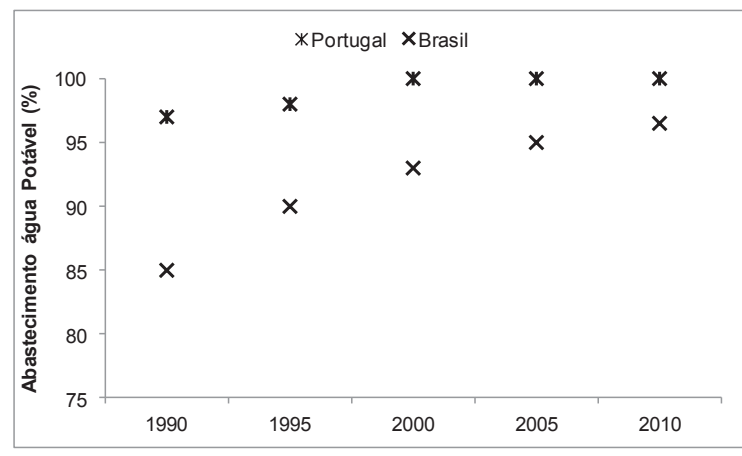

Fonte: Who (2011).

No território português, de acordo com os gráfi$\cos 2$ e 3, desde a década de 1990 ocorreu um aumento do acesso aos serviços de saneamento básico, com taxas próximas de $100 \%$ da população atendida.

Entretanto, a oferta e o acesso a serviços de saneamento básico, demonstram que o Brasil ainda está distante da equidade, como pode ser observado nos gráficos 2 e 3 . Ressaltam as diferenciações intra-regionais, com predomínio do acesso a esses serviços no SulSudeste. Entretanto, as doenças relacionadas com a água, se manifestam em todas as regiões brasileiras com diferentes intensidades.

Deve lembrar-se que a saúde incorpora a dimensão social, económica e cultural, por isso, o investimento em saneamento básico é diretamente um investimento na melhoria das condições de vida e da saúde da população.

As diferenças entre os territórios não se restringem ao clima, mas, como foi dito anteriormente, também incorporam o investimento em saúde e as desigualdades sociais.

É necessário criar condições para reduzir os riscos em ambos os territórios. Para isso, incorporar o clima como mais um instrumento de prevenção aos riscos para a saúde humana é absolutamente necessário.

Além disso, a participação em programas como as Cidades Saudáveis e outros que vêm reforçar a busca pela promoção da saúde, que no território Português, conta com uma rede estabelecida e pode ser um modelo para se estudar, pensar e planear a cidade saudável no Brasil, mesmo que sob diferentes proposições iniciais, uma vez que é preciso resolver carências básicas em algumas regiões brasileiras.

No entanto, é uma alternativa para que não ocorra a crise dentro do complexo de riscos no território e

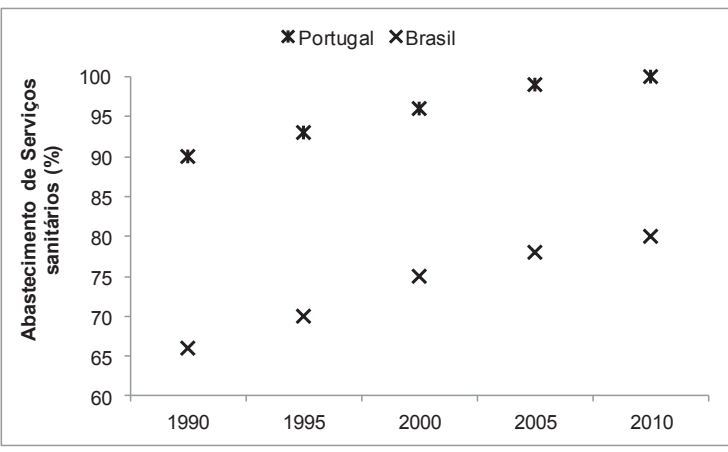

se consigam reduzir os espaços de vulnerabilidade, aumentando os espaços saudáveis.

\section{Considerações Finais}

O clima tropical, como um dos condicionantes para se pensar a vulnerabilidade da população e analisar o risco vivenciado, é um instrumento importante. Deve-se aprofundar o seu conhecimento, porém, livres de preconceitos que em outras épocas estiveram arreigados nos pesquisadores, devido ao chamado determinismo climático.

Sabe-se que muitas doenças que acometem os seres humanos são influenciadas, de forma direta ou indireta, pelas condições do tempo e do clima, mas é preciso que se pense o clima como um produto social, que é vivido e interpretado subjetivamente pela sociedade e é um dos condicionantes para ocorrência de diferentes grupos de doenças.

O clima pode e deve, assim, ser utilizado nas práticas de planeamento e na prevenção dos serviços de saúde para diminuição dos riscos de saúde humana.

Além disso, verificou-se que as condições de vulnerabilidade socioambiental e de gestão dos fatores de riscos oferecem capacidade de resiliência diferenciada à população em ambos os territórios.

Políticas públicas como a das "cidades saudáveis", precisam ser pensadas para o contexto brasileiro, a fim de se reduzir a vulnerabilidade da população. É preciso que o crescimento económico se traduza em desenvolvimento social, com melhorias na oferta e qualidade dos serviços de saúde, da educação pública, saneamento básico, entre outros aspetos necessários, para a maior participação social, bem-estar e qualidade de vida da população. 


\section{Referências Bibliográficas}

Albuquerque, M. B. M.; Lima, F. H. A. e Silva, T. A. O. C. (1999) "Doenças tropicais: da ciência dos valores à valorização da ciência na determinação climática de patologias". Ciência e Saúde Coletiva. 4(2), pp. 423-431.

Aleixo, N. (2008) - A influência do ritmo climático e da combustão da biomassa sobre a gênese da morbidade respiratória no espaço urbano de Ourinhos/SP. Monografia de conclusão do curso de bacharelado em Geografia, Universidade Estadual Paulista, Ourinhos, 2008.

AlmendRA, R. (2010) - Geografia da doença cardiovascular: Enfarte agudo do miocárdio - padrões e sazonalidade. Dissertação de mestrado em Geografia da Universidade de Coimbra, Coimbra/Portugal.

Andrade, H.; Nogueira, H. e CanárIo, P. (2011) - "Vulnerabilidade urbana ao calor extremo na área metropolitana de Lisboa”. Actas. VIII Congresso da Geografia Portuguesa, Lisboa.

Barros, M. (2006) - "Climas e endemias tropicais". Revista de Estudos Avançados. 20(58), pp. 297-306.

Basalla, G. (1997) - "La difusion de la ciencia occidental. Cuadernos del seminario". 3(1/2), pp. 1-20.

CAmargo, E. P. (2008) - "Doenças tropicais". Revista de Estudos avançados. 22(64). pp. 95-110.

Confalonieri, U. E. C. (2002) - "A crise ambiental e suas repercussões na saúde humana”. In: MinAYo, M. C. e Miranda, A. C. (org.) - Saúde e ambiente sustentável: estreitando nós. Rio de Janeiro: ABRASCO/FIOCRUZ. pp. 51-54.

Carvalho, V. F. M. (2007) - Contributos Bioclimáticos para o Planeamento Urbano Sustentável. Dissertação de Mestrado em Planejamento e Projecto do Ambiente Urbano, Universidade do Porto, Porto/Portugal.

Cunha, L. (2012) - "Riscos climáticos no Centro de Portugal. Uma leitura geográfica”. Revista Geonorte, ed. especial, Manuas, 4 (4), p. 105-115.

Dear, R. J.; Kalma, J. D. e OKe, T. R. (1999) - "Biometeorology and Urban Climatology at the turn of the Millennium". Actas. WCASP 50 WMO/TD. pp. 34-48.

DrIsColl, D. M. (1999) - "Some directions for human biometeorology in the new century". WCASP 50. WMO/ TD. pp.73-77.

Ferreira, M. E. M. C. e Lombardo, M. A. (2000) - "A variabilidade climática e a ocorrência de malária na área de influência do reservatório de Itaipu - Paraná, Brasil”. In: SANT'ANNA Neto, João Lima (org.) - Variabilidade e mudanças climáticas. Implicações ambientais e socioeconômicas. EDUEM-UEM, Maringá/PR, pp. 147-163.

ForatinnI, O. P. (1997) - "O Brasil e a medicina tropical". Revista de Saúde Pública. 31(2), pp. 116-120.
Frota, A. B. e SchifFER, S. R. (2003) - Manual de conforto térmico. Studio Nobel, São Paulo.

Galvani, E. (2005) - "Considerações acerca dos estudos bioclimáticos". In: Anais do X Encontro de Geógrafos da América Latina. Universidade de São Paulo, São Paulo/SP.

Gonçalves, F. L. T. e KRIEger, S. (2004) - Estudos biometeorológicos do clima urbano. Instiruto de Astrofísica da Universidade de São Paulo, São Paulo.

Gonçalves, A. J. B; Lourenço, L. e Silva, J. D. (2007) - Manifestação dos incêndios florestais em Portugal continental, causas e investigação criminal. Territorium, Revista da Associação Portuguesa de Riscos, Prevenção e Segurança, Lousã, $n^{\circ} 14$, pp. 81-87.

HoPPE, P. (1997) - "Aspects of human biometeorology, past, present and future". Internacional Journal of Biometeorology. vol. 40, pp.19-23.

JAUREGUI, E. (1999) - "Tropical Urban Climatology at the turn of the millennium - An overview". Biometeorology and Urban Climatology at the turn of the Millennium-WCASP 50. WMO/TD, pp. 537-544.

JAUREGUI, E. (1994) - "Overwiew of papers on urban climate in tropical/subtropical areas 1981-1991". Report of the technical conference on tropical urban climate. $\mathrm{n}$. 647, pp.377-390.

JendritZky, E.; Grätz , A. e Schultz, E. (1994) - "Endlicher W. Urban Bioclimatology". In: Report of the technical conference on tropical urban climates. Dhaka, Blangladesh. WCASP 30. WHO/TD, n.647, pp.245-264.

LouRenço, L. (2006) - "Incêndios florestais. Algumas reflexões sobre prevenção e mitos do combate". Territorium, Revista da Associação Portuguesa de Riscos, Prevenção e Segurança, Lousã, n 13, pp. 59-70.

Lourenço, L.; Gonçalves, A. B. e Bento, M. S. (2001) - "Intervenção humana e risco de fogo florestal". Cadernos de Geografia, n. ${ }^{\circ}$ especial, pp. 91-98.

Marques, J e Antunes, S. (2009) - "A perigosidade natural da temperatura do ar em Portugal continental: A avaliação do risco na mortalidade". Revista Territorium, n. 16, pp. 49-63.

Mendes, V. C. (2009) - Ondas de calor e conforto térmico: Caracterização de lares de idosos, centros de saúde e hospitais da região centro face a ocorrência de fenômenos climáticos intensos. Dissertação de Mestrado em Geografia, Universidade de Coimbra, Coimbra/ Portugal.

Mendonça, F. (2000) - "Aspectos da interação clima - ambiente saúde humana: Da relação sociedade-natureza à (in) sustentabilidade ambiental”. Revista RA'E GA, vol.4, n. ${ }^{\circ} 4$, pp. 85-100.

Mendonça, F. e PaUla, E. V. (2002) - "Análise geográfica da dengue no Paraná e em Curitiba no período 1995-2002: um 
enfoque climatológico". Actas. V Simpósio Brasileiro de Climatologia Geográfica, pp.1-15.

Mendonça, F. A.; Paula, E. V. e Oliveira, M. M. F. (2006) - "Aspectos socioambientais da expansão da dengue no Paraná". In: Jacobi, Pedro e Ferreira, Lucia da Costa. (org.) - Diálogos em ambiente e sociedade no Brasil. Annablume, São Paulo, vol. 1, pp. 329-342.

Moran, F. (1994) - Adaptabilidade Humana. Edusp. São Paulo. Monteiro, A. (1993) - O clima urbano do Porto. Contribuição para definição de estratégias de de Planeamento e Ordenamento do Território. Tese de doutoramento, Universidade do Porto, Porto/Portugal.

Montelro, A. (1997) - "Clima-Qualidade do ar-Saúde: um nexo de causalidade (in)desejável para o Homem?". Revista da Faculdade de Letras, I Série, vol.XII/XIII, pp. 39-71.

Monteiro, A. (2001) - "Os impactos do clima na qualidade do ar e na saúde, enquanto potenciais indicadores de ausência de estratégia de desenvolvimento de um espaço urbano: Estudo de caso na área metropolitana do Porto". Actas. VII Conferência sobre a Qualidade do Ambiente, Aveiro, pp.851-869.

Murara, P. G. e Amorim, M. C. C. T. (2010) - "Clima e Saúde: variações atmosféricas e óbitos por doenças do aparelho circulatório". Revista brasileira de climatologia, vol. 6, pp. 79-92.

Peixoto, A. (1938) - Clima e saúde. Cia. Editora Nacional, São Paulo.

Pitton, S. E. e Domingos, A. E. (2004) - "Tempos e doenças: efeitos dos parâmetros climáticos nas crises hipertensivas nos moradores de Santa Gertrudes - SP". Revista de Estudos Geográficos. vol. 02, nº 01, pp.75-86.
ReBeLo, F. (2001) - Riscos Naturais e acção antrópica. Imprensa da Universidade de Coimbra, 2001.

Ribeiro Sobral, H. (1996) - Ilha de calor na cidade de São Paulo: sua dinâmica e efeitos na saúde da população. Tese de Livre-docência da FSP/USP, São Paulo.

RibeiRo, H. (2005) - "Heat Island in São Paulo, Brazil: effects on health". Critical Public Health, v. 15, n. 2, pp. 147-156.

RibeiRo, H. (1988) - Poluição do ar e doenças respiratórias em criança da Grande São Paulo: um estudo de geografia médica. Tese de Doutorado em Geografia. Universidade de São Paulo, São Paulo.

Sant Anna Neto, J. L. (2004) - "História da climatologia no Brasil”. Cadernos Geográficos, n. ${ }^{\circ}$ 7, pp. 10-57.

SILVA, E. N. (2010) - Ambientes atmosféricos intraurbanos em São Paulo e possíveis correlações com doenças do aparelho respiratório e circulatório. Tese de doutorado em Saúde Pública. Universidade de São Paulo, São Paulo.

SORRE, M. (1984) - "A adaptação ao meio climático e biossocial - geografia psicológica". In: MEGALE, J. F. (org.) Coleção Grandes cientistas sociais. Ática, São Paulo.

SouzA, C.G. (2007) - A influência do ritmo climático na morbidade respiratória em ambiente urbano. Dissertação em Geografia, FCT/UNESP:Presidente Prudente.

VILLA, T. C. S. (2009) - "Estratégias de pesquisa para o controle de doenças negligenciadas: projetos colaborativos de enfermagem em rede". Revista Latino-Americana de Enfermagem; 17(4), pp. 437-8.

World Health Organization (2010) - Neglected tropical diseases. Disponível em: www.who.int/neglected-disease/en/. Acesso: 29 de julho de 2011. 